\title{
Bioactivity of the Vascular Endothelial Growth Factor Trapped in Fibrin Clots: Production of IL-6 and IL-8 in Monocytes by Fibrin Clots
}

\author{
Kazuhito Tezono ${ }^{a} \quad K^{\prime}$ rishna Pada Sarker ${ }^{b}$ Hirosi Kikuchi ${ }^{a}$ \\ Masaru Nasua $^{a}$ Isao Kitajimab Ikuro Maruyamab \\ a Second Department of Internal Medicine, Oita Medical University, Oita, and \\ bDepartment of Laboratory and Molecular Medicine, Kagoshima University School of \\ Medicine, Kagoshima, Japan
}

\section{Key Words \\ VEGF · Fibrin clot · Endothelial monocytes · \\ Cytokines - Wound healing}

\begin{abstract}
The blood coagulation cascade is activated following vascular-wall injury. The serine protease thrombin is the final protease in this cascade that causes the formation of fibrin from fibrinogen. Thrombin also causes the activation of platelets, which are trapped in a fibrin net followed by hemostasis. Platelets gathered into fibrin clots release several growth factors such as platelet-derived growth factor and transforming growth factor $\beta$. In the present study, we demonstrated that the vascular endothelial growth factor (VEGF) could be bound to fibrin clots in the plasma, and that incubation of the endothelial cells with these VEGF-bound fibrin clots
\end{abstract}

induced proliferation of endothelial cells. Thus, it suggests that clot-bound VEGF may play a role in wound healing through the proliferation of endothelial cells and vascular smooth-muscle cells. On the other hand, a noticeable migration of monocytes was observed when they were cultured on dishes in the presence of VEGF-bound fibrin clots. Moreover, peripheral blood monocytes incubated in the presence of VEGF-bound fibrin clots strikingly increased the production of IL-6 and IL-8, demonstrating that VEGF trapped in fibrin clots not only induces proliferation of human umbilical vein endothelial cells and migration of monocytes but also enhances secretion of IL- 6 and IL-8. Thus, our data suggest that fibrin clots that contain several growth factors act as a bioactive reservoir and may play an important role in hemostasis as well as wound healing.

Copyright @ 2001 S. Karger AG, Basel

Dr. Ikuro Maruyama

Department of Laboratory and Molecular Medicine

Kagoshima University School of Medicine, 8-35-10 Sakuragaoka Kagoshima 890-8520 (Japan), Tel. +81 992755437

Fax +8199275 2629, E-Mail rinken@khosp2.kufm.kagoshima-u.ac.jp 


\section{Introduction}

Vascular endothelial growth factor (VEGF) is a homodimeric glycoprotein with a potent mitogenic activity on endothelial cells. VEGF also enhances vascular permeability and thus is also called VEGF/VPF (vascular permeability factor) [1-3]. VEGF is encoded by a single gene, which finally produces four isoforms, VEGF-121, VEGF-165, VEGF-189 and VEGF-206 (based on the number of amino acids) generated by posttranscriptional modification [4, 5]. VEGF-121 and VEGF165 are present in the circulation, VEGF-189 and VEGF-206 remain on the cell membrane bound to glucose aminoglycans. VEGF-165, VEGF-189 and VEGF-206 possess heparinbinding sites, whereas VEGF-121 does not [6-8]. It is well established that VEGF plays a pivotal role in normal vessel growth, development, differentiation, wound healing and reproduction. Furthermore, VEGF has potent mitogenic and chemotactic activity on endothelial cells and acts as an angiogenic factor [1-3]. The blood coagulation cascade is induced following vessel wall injury, which triggers the activation of thrombin. Once thrombin is activated, it cleaves fibrinogen into fibrin. Platelets are gathered into fibrin clots which may play a key role in hemostasis.

This study was designed to investigate whether VEGF could be trapped in fibrin clots and would exert its characteristic bioactivity on endothelial cells as well as monocytes in vitro. We show the evidence of entrapment/binding of VEGF to fibrin clots. Clotbound VEGF acted as a growth factor for endothelial cells. Moreover, VEGF trapped in fibrin clots acted as a chemotactic factor for monocytes and induced IL-6 and IL- 8 production in those cells, suggesting the bioactivity of VEGF-bound fibrin clots.

\section{Material and Methods}

Materials

Human thrombin was obtained from Mochida Pharmaceutical Co. Ltd. (Tokyo, Japan). VEGF-165 was purchased from R \& D Co. (Minneapolis, Minn., USA). Anti-VEGF antibody was obtained from Toagousei Co. (Tsukuba, Japan) [9, 10]. Fibrinogen and other reagents were purchased from Sigma (St. Louis, Mo., USA).

\section{Cell Culture}

Human umbilical vein endothelial cells (HUVECs) were prepared and subjected to primary culture, as described [11]. Human peripheral blood monocytes were collected from healthy volunteers as described $[12,13]$.

\section{Immunostaining of VEGF}

The microsections of clots prepared from normal human whole blood was immunostained. Immunostaining was performed by direct immunoperoxidase staining as described [14]. Briefly, 4-mm paraffinembedded specimens were deparaffinized in xylene, followed by rehydration with ethanol $(100,95,70$ and $50 \%$ ). To inhibit the reaction of endogenous peroxidase, the sections were immersed in methanol-containing $0.001 \% \mathrm{H}_{2} \mathrm{O}_{2}$ for $20 \mathrm{~min}$ and allowed to airdry.

\section{VEGF Measurement}

VEGF concentration was measured by a newly developed colorimetric enzyme-linked immunosorbent assay, as described [15-17]. Briefly, the antiVEGF/VPF IgG was used as a plate coating and a peroxidase-conjugated Fab fragment of anti-human IgG was used as a secondary antibody. Microtiter plates (Immulon 2, Dynatech, USA) were coated with antiVEGF/VPF polyclonal antibody against human VEGF-121, which can detect all isoforms of VEGF at a concentration as low as $20 \mathrm{pg} / \mathrm{ml}$ in serum or plasma. Absorbance at $490 \mathrm{~nm}$ was measured in a plate reader after reacting for $30 \mathrm{~min}$.

Preparation and Standardization of Fibrin Clots

Fibrin clots were prepared by adding VEGF-165 into purified fibrinogen, followed by thrombin. To standardize the fibrin clots, an equal volume of VEGF165 (final VEGF-165 concentration $100 \mathrm{pg} / \mathrm{ml}$ ) was added to the purified fibrinogen. Then, $1 \mathrm{ml}$ of VEGFcontaining fibrinogen solution was filled into each tube, and thrombin was added to it, which led to clot formation. Fibrin clots were carefully washed three times in phosphate-buffered saline before use. $\overline{72} \quad \overline{\text { Haemostasis 2001;31:71-79 }}$
Tezono/Sarker/Kikuchi/Nasu/ Kitajima/Maruyama 
Detection of VEGF Entrapment in Fibrin Clots

Radiolabeled VEGF was prepared by adding VEGF (to a final concentration of $100 \mathrm{pg} / \mathrm{ml}$ ) to the iodogen ${ }^{125}$ I. The radiolabeled VEGF ( ${ }^{125}$ I-VEGF) was added to normal human plasma or a euglobulin fraction [18] prepared from normal plasma. Fibrin clots were generated by adding thrombin $(10 \mathrm{U} / \mathrm{ml})$ to plasma or a euglobulin fraction that contained ${ }^{125}$ I-VEGF. The radioactivity corresponding to the existence of VEGF in the supernatant as well as in the clot lysate was counted by a scintillation counter.

Cell Proliferation Assay

A 3-(4,5-dimethyl-2-thiazolyl)-2,5-diphenyl-2H-tetrazolium bromide (MTT) assay was performed to test the proliferation of cells in response to clot-bound VEGF. Briefly, cells were seeded at a density of 2.0$2.5 \times 10^{4} \mathrm{cells} /$ well onto 96-well dishes and were incubated with the fibrin clots for the indicated periods. Following this incubation, MTT $(0.5 \mathrm{mg} / \mathrm{ml}$ final concentration) was added to each well. As mitochondrial enzyme converts MTT to insoluble formazan crystal, $100 \mu \mathrm{l}$ of a solution containing $10 \% \operatorname{SDS}(\mathrm{pH} 4.8)+$ $0.01 \mathrm{NHCl}$ was added to dissolve the crystal $3 \mathrm{~h}$ after the addition of MTT. Absorbance values were determined with an automatic microtiter plate reader (Immuno Mini NJ-2300, Japan) at 570-630 nm.

Assay of IL-6 and IL-8

Cytokines (IL-6 and IL-8) were measured in the supernatant of cultured monocytes, as described [19].

\section{Results}

In this study, we investigated whether VEGF was entrapped in fibrin clots in vitro. We prepared blood clots from normal human whole blood letting it stand in a glass tube in the presence of thrombin $(10 \mathrm{U} / \mathrm{ml})$ at room temperature. Microsections from the clots were subjected to VEGF immunostaining, and the existence of immunoreactive VEGF in clots was confirmed by immunohistochemical methods (fig. 1). Positive staining of VEGF was observed lineally on the surface of the clot (fig. 1B, thick arrow). Positive staining was also observed in the mononuclear cells (fig. 1B, thin arrow) and fibrous portion of the inner part of the clot (fig. 1B and D, broken arrows), indicating the presence of VEGF not only on the clot surface but also inside the clots. Thus, positive staining in the fibrous portion demonstrated the association of VEGF with fibrin clots.

To further confirm the binding to and entrapment of VEGF in clots, especially its association with fibrin, we performed an entrapment study using radiolabeled VEGF, which exhibited the same biological activities as untreated VEGF (data not shown). Although we have no direct evidence that VEGF bound to fibrin, the radioactivity of ${ }^{125} \mathrm{I}-\mathrm{VEGF}$ reflecting the concentration of VEGF in the supernatant of the plasma-derived fibrin clots was decreased compared to that of the plasma before the clot formation (fig. 2, column 3), implying the entrapment of ${ }^{125}$ I-VEGF in the clot. As clots formed from normal plasma were resistant to fibrinolysis because of the contamination with $\alpha_{1}$-antiplasmin, we prepared plasmin-sensitive fibrin clots from a euglobulin fraction (plasmin inhibitor deficient). Similarly to plasma, the radioactivity of the euglobulin fraction had been decreased after the clot formation (fig. 2, column 5), further supporting the binding of VEGF to fibrin clots. Then, the radioactivity of a clot-lysed solution prepared by plasmin digestion in vitro was assessed. We observed that more than $80 \%$ of ${ }^{125}$ I-VEGF appeared in the clot-lysed supernatant as the radioactivity increased (fig. 2, column 6), suggesting that VEGF was entrapped in the fibrin clots.

Next, we investigated the biological activity of the clots, which were prepared using various concentrations of VEGF added to fibrinogen. The number of cells increased with the increasing amount of VEGF-containing fibrinogen. As shown in figure 3 , the addition of the clot containing $10 \mathrm{pg} / \mathrm{ml}$ VEGF to HUVECs increased the cell-proliferating activity, which then increased significantly 

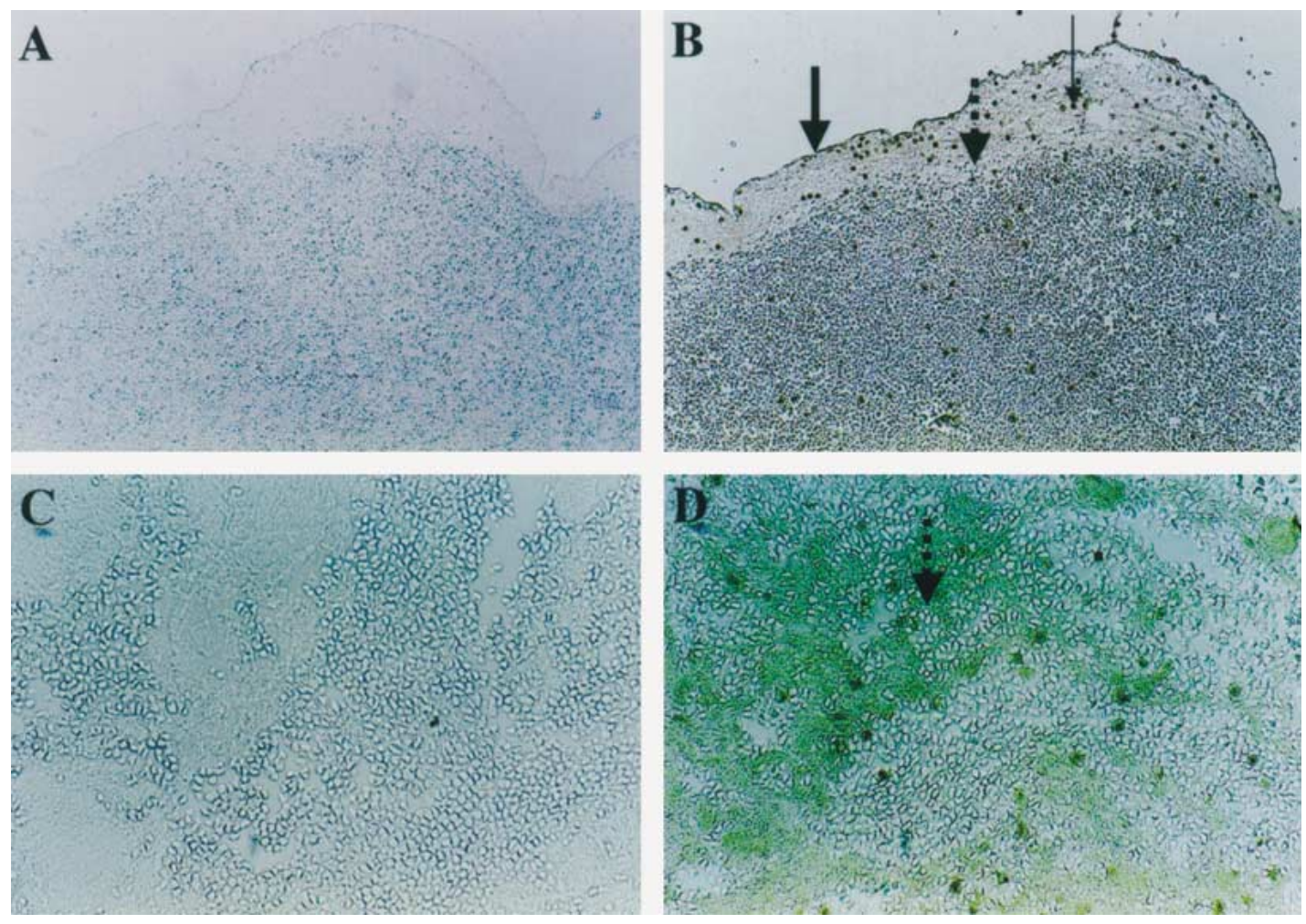

Fig. 1. Immunostaining of VEGF in fibrin clots. Fibrin clots were formed by addition of thrombin $(10 \mathrm{U} / \mathrm{ml})$ in vitro. A Anti-human $\mathrm{IgG}$ antibody $\times 85$. B Anti-human VEGF antibody $\times 85$. C Anti-human IgG $\times 350$. D Anti-human VEGF antibody $\times 350$. Thick arrow $=$ Surface of the clot; thin arrow $=$ mononuclear cell in the fibrin clot; broken arrows $=$ fibrous portion of the inner part of the clot. The photographs are representative of three independent experiments.

when cells were plated with fibrin clots containing $1,000 \mathrm{pg} / \mathrm{ml}$ VEGF. The addition of antibody to VEGF $(100 \mathrm{ng} / \mathrm{ml})$ ceased this cell proliferation, suggesting that the cell growth was induced by VEGF (fig. 3). However, the anti-VEGF could not completely block the proliferating activity of fibrin clots, suggesting that the neutralizing activity of the antibody may be insufficient to block the bioactivity of fibrin-bound VEGF or may not have entered the clots properly.
As VEGF induces various biological effects on monocytes, we further examined the ability of fibrin-clot-bound VEGF to induce migration of monocytes. Equal numbers of monocytes $\left(2.5 \times 10^{4}\right.$ per well $)$ were plated onto 96-well dishes and cultured in the presence or absence of fibrin clots. It was observed that monocytes markedly migrated towards fibrin clots (fig. 4B), indicating that VEGF trapped in fibrin clots was able to act as a chemoattractant to monocytes.

\section{4}

Haemostasis 2001;31:71-79
Tezono/Sarker/Kikuchi/Nasu/ Kitajima/Maruyama 
Fig. 2. Release of VEGF in fibrinclot-lysed supernatant. $1=$ Control (normal plasma); 2 = radioactivity $(\mathrm{cpm} / \mathrm{ml})$ of the normal human plasma after addition of ${ }^{125} \mathrm{I}$ VEGF; 3 = radioactivity of the supernatant after the formation of fibrin clots by the addition of thrombin $(10 \mathrm{U} / \mathrm{ml})$ to the plasma (reduction of radioactivity suggesting the binding of radiolabeled VEGF to the clot); $4=$ radioactivity of the euglobulin fraction (plasmin-inhibitor-deficient) after the addition of ${ }^{125}$ I-VEGF; 5 = radioactivity of the supernatant after fibrin clot formation by the addition of thrombin $(10 \mathrm{U} / \mathrm{ml})$ to the euglobulin fraction; $6=$ radioactivity of the clotlysed supernatant (prepared using an euglobulin fraction). Data represent means \pm SD of three separate experiments.

Fig. 3. Effect of VEGF bound to fibrin clots on the proliferation of HUVECs. HUVECs were plated on 96-well dishes and cultured at various concentrations of VEGF in fibrin clot in the presence or absence of antibody of VEGF (100 $\mathrm{ng} / \mathrm{ml}$ ) for $72 \mathrm{~h}$. Data represent means \pm SD of three separate experiments. Cell proliferation was assessed afterwards by MTT assay.
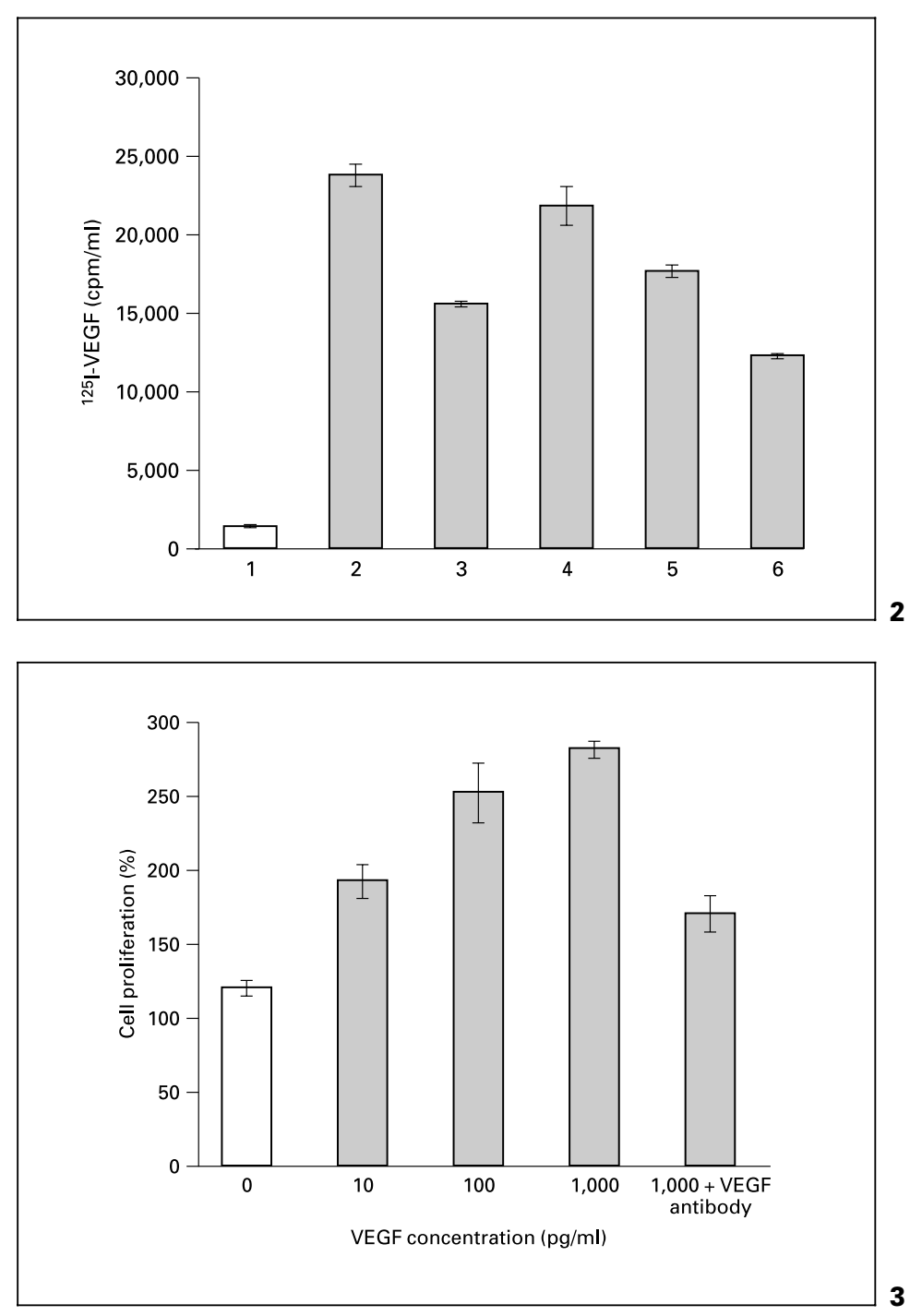

Finally, we assayed the concentrations of IL-6 and IL-8 in the culture of monocytes. VEGF bound to fibrin clots significantly enhanced the production of IL- 6 and IL- 8 in vitro. In response to VEGF, the production of IL-6 was significantly increased to $1,095 \pm$ $13 \mathrm{pg} / \mathrm{ml}$; it decreased to $47 \pm 2 \mathrm{pg} / \mathrm{ml}$ when treated with $100 \mathrm{ng} / \mathrm{ml}$ anti-VEGF. Similarly,
VEGF significantly increased the secretion of IL-8 to $18,714 \pm 584 \mathrm{pg} / \mathrm{ml}$; this decreased to $3,127 \pm 324 \mathrm{pg} / \mathrm{ml}$ in the presence of antiVEGF, demonstrating that clot-bound VEGF stimulated peripheral monocytes to produce IL-6 and IL-8 (fig. 5). 

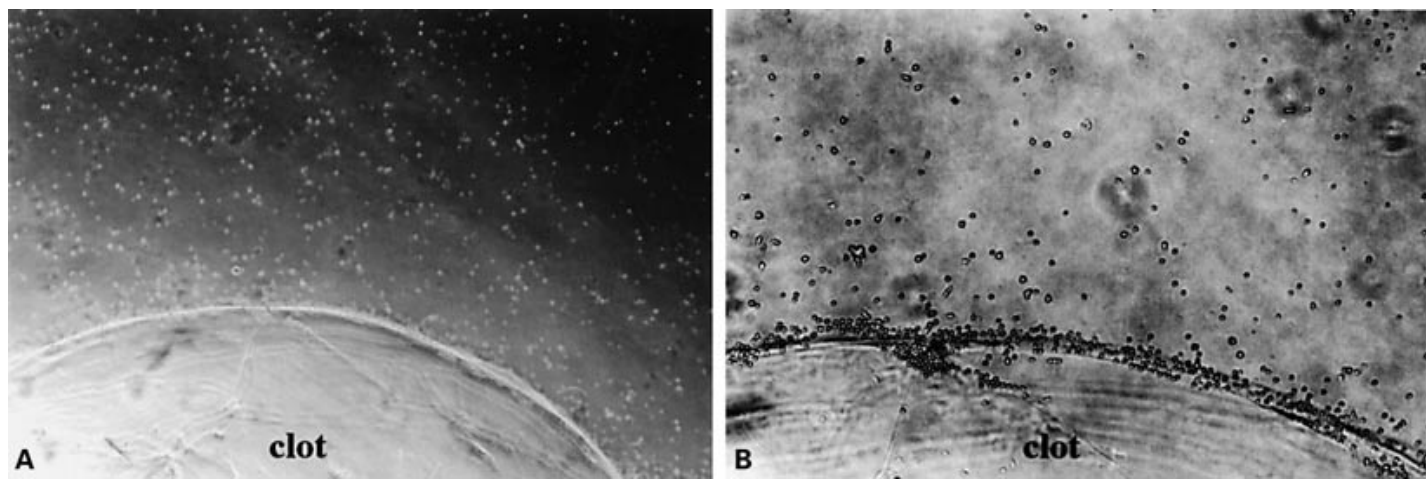

Fig. 4. Effect of VEGF bound to fibrin clots on cultured monocytes. Peripheral monocytes were cultured on 96-well dishes in the presence or absence of VEGF (1,000 pg/ml). Migration and adherence of monocytes were observed by light microscopy. Photographs were taken after $24 \mathrm{~h}$ of fibrin clot addition. A Control monocytes (fibrin clot without VEGF). B Fibrin clot containing VEGF. Duplicate experiments were also done. Magnification $\times 180$.

\section{Discussion}

When blood vessels are injured, a prompt blood coagulation cascade is initiated resulting in the generation of thrombin. Thrombin generated in this way activates platelets, which play an important role in the formation of hemostatic thrombi, followed by the repair of the injured vessels. During the repairing process, the proliferation of vessel wall cells is indispensable. It has been demonstrated that platelet-derived growth factor (PDGF) released by the activated platelets plays an important role in this cellular event. PDGF acts as a chemotactic and proliferating factor mainly on vascular smooth-muscle cells and vascular fibroblasts [20, 21]. On the other hand, the proliferation of endothelial cells is essential for the wound repairing process. It has been reported that VEGF acts chemotactically and mitogenically on endothelial cells. It has also been reported that the mitogenic activity of VEGF on endothelial cells is much greater than that of PDGF [22, 23].
In the present study, we first investigated the possibility of trapping VEGF into clots in vitro. Human plasma and serum contain 0$10 \mathrm{pg} / \mathrm{ml}$ and $80-100 \mathrm{pg} / \mathrm{ml}$ VEGF, respectively [24]. Our preliminary results indicate that more than $100 \mathrm{pg} / \mathrm{ml}$ of VEGF are required for it to act mitogenically on endothelial cells. However, as shown in figure 3, fibrin-clot-bound VEGF exhibited a very potent cell-proliferating activity on cultured HUVECs. The incubation of the HUVEC monolayers with VEGF-containing fibrin clots, which were prepared with fibrinogen containing as little as $10 \mathrm{pg} / \mathrm{ml}$ of VEGF showed cell proliferation activity (fig. 3). Thus, the clot surface VEGF appeared to be more active than that of the fluid; however, further studies are needed to confirm this. We did not observe a linear dose-responsive effect of VEGF-containing fibrin clots on cell-proliferating activity (fig. 3). This may be due to the fact that VEGF on the clot surface does not correlate exactly with the VEGF content of the fibrinogen solution. Of note, we tried to $\overline{76} \quad \overline{\text { Haemostasis 2001;31:71-79 }}$
Tezono/Sarker/Kikuchi/Nasu/ Kitajima/Maruyama 
Fig. 5. Fibrin clot having VEGF induces production of the cytokines IL-6 and IL-8 on monocytes. Peripheral monocytes were cultured with VEGF $(1,000 \mathrm{pg} / \mathrm{ml})$ in the presence or absence of antibody to VEGF $(100 \mathrm{ng} / \mathrm{ml})$. Cytokines were measured at $24 \mathrm{~h}$ after fibrin clot addition in the supernatant of cultured monocytes. A Level of IL6. B Level of IL-8. Data represent means \pm SD of three independent experiments.
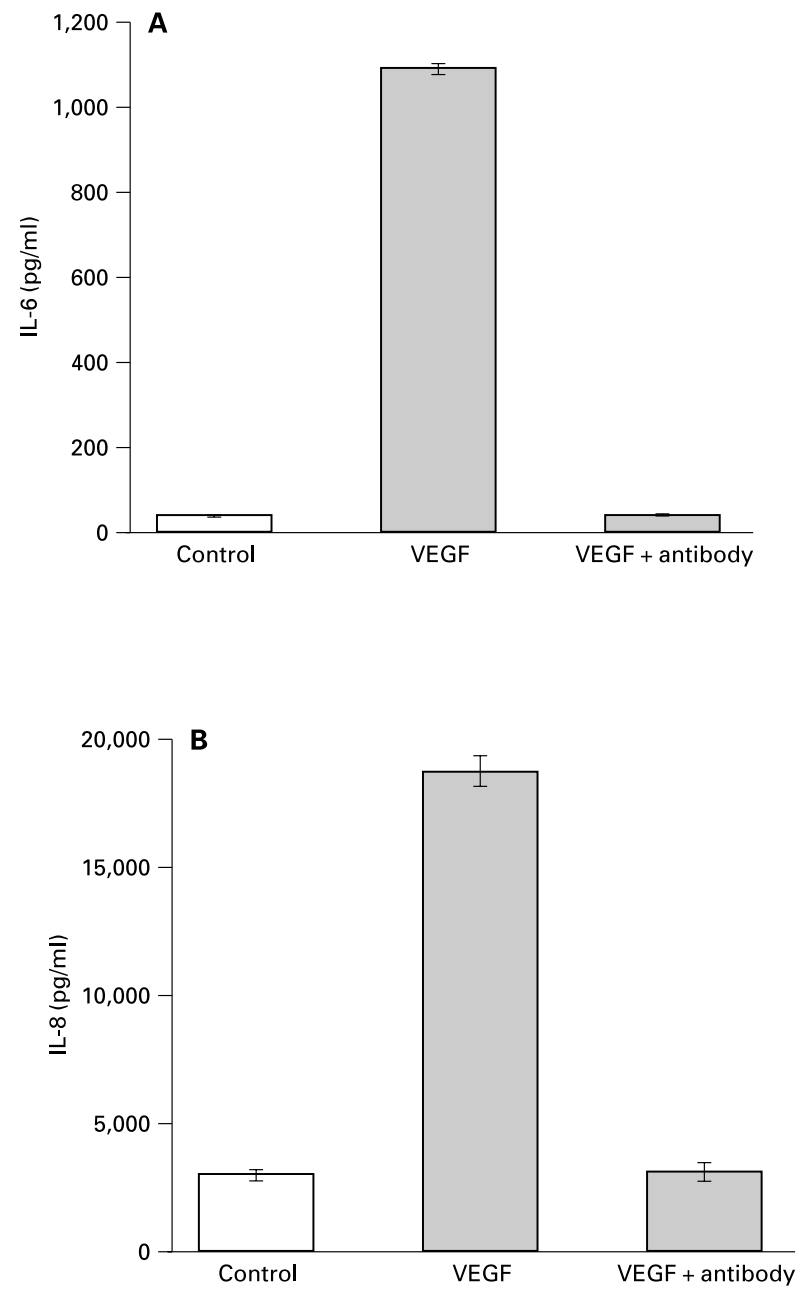

prepare fine fibrin clots by sonication but failed. Hence, we used fibrin clots per se in the experiments. We obtained $57 \%$ of VEGF in the lysate of clots obtained by plasmin digestion, demonstrating the existence of VEGF in the clots. Our result is also in line with the recent finding that VEGF binds to fibrinogen and that this VEGF-fibrinogen complex is present in the circulation [25]. However, VEGF also originates from platelets and is stored in the clots in vivo. A number of cells synthesize VEGF in response to mitogens in vitro. We as well as others have also reported that thrombin enhances the release of VEGF 
in the culture medium and that the mitogenic activity of thrombin may be dependent in part on VEGF. VEGF is synthesized by megakaryocytes and also contained in the circulating platelets [26-28]. Thus, we formed the hypothesis that VEGF can be released from platelets upon stimulation and may be trapped and stored in the thrombus. Our result shows that fibrin clots induce the proliferation of HUVECs in vitro, suggesting that clotbound VEGF has a potent mitogenic activity. It has also been described that VEGF exerts several biological activities on peripheral monocytes such as chemotaxis, tissue factor expression and influx of intercellular $\mathrm{Ca}^{2+}$ [28]. We demonstrated that fibrin clots stimu- lated the monocytes and induced the release of IL-6 and IL-8. Thus, clot-bound VEGF may act on peripheral monocytes.

In summary, we propose a hypothesis based on our present data that blood coagulation would start at the site of vessel injury, resulting in hemostatic thrombus formation, where platelets also adhere and aggregate with the secretion of VEGF. The secreted VEGF may be stored and enriched in the clots, which may provide a proper environment to wound healing promoting endothelial chemotaxis and growth. Thus, clot-bound VEGF appears to be a kind of bioactive reservoir and 'scaffold' to wound repair.

\section{References}

1 Ferrara N, Winer J, Burton T: Aortic smooth muscle cells express and secrete vascular endothelial growth factor. Growth Factors 1991;5:141148.

2 Kuzuya M, Satake S, Esaki T, Yamada K, Hayashi T, Naito M, Asai $\mathrm{K}$, Iguchi A: Induction of angiogenesis by smooth muscle cell-derived factor: Possible role in neovascularization in atherosclerotic plaque. J Cell Physiol 1995;164:658-667.

3 Sunderkotter C, Steinbrink K, Goebeler M, Bhardwaj R, Sorg C: Macrophages and angiogenesis. J Leukoc Biol 1994;55:410-422.

4 Tischer E, Mitchell R, Hartman T, Silva M, Gospodarowicz D, Fiddes JC, Abraham JA: The human gene for vascular endothelial growth factor. Multiple protein forms are encoded through alternative exon splicing. J Biol Chem 1991;266: 11947-11954.
5 Charnock Jones DS, Sharkey AM, Rajput-Williams J, Burch D, Schofield JP, Fountain SA, Boocock CA, Smith SK: Identification and localization of alternately spliced mRNAs for vascular endothelial growth factor in human uterus and estrogen regulation in endothelial carcinoma cell lines. Biol Reprod 1993;48:1120-1128.

6 Conn G, Soderman DD, Schaeffer MT, Wile M, Hatcher VB, Thomas KA: Purification of a glycoprotein vascular endothelial cell mitogen from a rat glioma-derived cell line. Proc Natl Acad Sci USA 1990;87: 1323-1327.

7 Park JE, Keller GA, Ferrara N: The vascular endothelial factor (VEGF) isoforms: Differential deposition into the subepithelial extracellular matrix and bioactivity of extracellular matrix-bound VEGF. Mol Biol Cell 1993;4:1317-1326.

8 Ferrara N, Davis-Smyth T: The biology of vascular endothelial growth factor. Endocr Rev 1997;18:4-25.

9 Asano M, Yukita A, Matsumoto T, Hanatani M, Suzuki H: An antihuman VEGF monoclonal antibody, MV833, that exhibits potent anti-tumor activity in vivo. Hybridoma 1998;17:185-190.
10 Asano M, Yukita A, Suzuki H : Wide spectrum of antitumor activity of a neutralizing monoclonal antibody to human vascular endothelial growth factor. Jpn J Cancer Res 1999;90:93-100.

11 Jaffe EA, Nachman RL, Becker CG, Minick CR: Culture of human endothelial cells derived from umbilical veins. Identification by morphologic and immunologic criteria. J Clin Invest 1973;52:2745-2756.

12 Koga M, Ishihara T, Takahashi M, Umezawa Y, Furukawa S: Activation of peripheral blood monocytes and macrophages in Kawasaki disease: Ultrastructural and immunocytochemical investigation. Pathol Int 1998;48:512-517.

13 Grage-Griebenow E, Lorenzen D, Fetting R, Flad HD, Ernst M: Phenotypical and functional characterization of $\mathrm{Fc}$ gamma receptor I (CD64)-negative monocytes, a minor human monocyte subpopulation with high accessory and antiviral activity. Eur J Immunol 1993;23: 3126-3135. 
14 Davidoff M, Schulze W: Combination of the peroxidase anti-peroxidase (PAP)- and avidin-biotin-peroxidase complex (ABC)-techniques: An amplification alternative in immunocytochemical staining. Histochemistry 1990;93:531-536.

15 Hanatani M, Tanaka Y, Kondo S, Ohmori I, Suzuki H: Sensitive chemiluminescence enzyme immunoassay for vascular endothelial growth factor/vascular permeability factor in human serum. Biosci Biotechnol Biochem 1995;59:19581959.

16 Miyata K: Increased circulating vascular endothelial growth factor is correlated with disease activity in polyarticular juvenile rheumatoid arthritis. J Rheumatol 1999;26: 2244-2248.

17 Sarker KP, Nakata M, Nakajima T, Kitajima I, Maruyama I: Increased production of vascular endothelial growth factor (VEGF) by angiotensin II in neuro-2a cells. Neurosci Res Commun 1999;25:79-88.

18 Kowalski E, Kopec M, Niewarowski $\mathrm{S}$ : Evaluation of a euglobulin method for determination of fibrinolysis. J Clin Pathol 1959;12:215-219.
19 Kita M, Ohmoto Y, Yamaguchi N, Imanishi J: Induction of cytokines in human peripheral blood mononuclear cells by mycoplasmas. Microbiol Immunol 1992;36:507-516.

20 Deuel TF, Huang JS: Platelet-derived growth factor. Structure, function, and roles in normal and transformed cells. J Clin Invest 1984;74: 669-676.

21 Heldin CH, Westermark B: Mechanism of action and in vivo role of platelet-derived growth factor. Physiol Rev 1999;79:1283-1316.

22 Connolly DT, Heuvelman DM, Nelson R, Olander JV, Eppley BL, Delfino JJ, Siegel NR, Leimgruber RM, Feder J: Tumor vascular permeability factor stimulates endothelial cell growth and angiogenesis. J Clin Invest 1989;84:1470-1478.

23 Keck PJ, Hauser SD, Krivi G, Sanzo K, Warren T, Feder J, Connolly DT: Vascular permeability factor, an endothelial cell mitogen related to PDGF. Science 1989;246:13091312.
24 Hashiguchi T, Arimura K, Matsumuro K, Otsuka R, Watanabe $\mathrm{O}$, Jonosono M, Maruyama Y, Maruyama I, Osame M: Highly concentrated vascular endothelial growth factor in platelets in Crow-Fukase syndrome. Muscle Nerve 2000;23: 1051-1056.

25 Sahni A, Francis CW: Vascular endothelial growth factor binds to fibrinogen and stimulates endothelial cell proliferation. Blood 2000;96: 3772-3778.

26 Mohle R, Green D, Moore MA, Nachman RL, Rafii S: Constitutive production and thrombin-induced release of vascular endothelial growth factor by human megakaryocytes and platelets. Proc Natl Acad Sci USA 1997;94:663-668.

27 Maloney JP, Silliman CC, Ambruso DR, Wang J, Tuder RM, Voelkel NF: In vitro release of vascular endothelial growth factor during platelet aggregation. Am J Physiol 1998; 275:1054-1061.

28 Banks RE, Forbes MA, Kinsey SE, Stanley A, Ingham E, Walters C, Selby PJ: Release of the angiogenic cytokine vascular endothelial growth factor (VEGF) from platelets: Significance for VEGF measurements and cancer biology. $\mathrm{Br} \mathrm{J}$ Cancer 1998;77:956-964. 